\title{
Influence of emotional state on irrelevant thoughts
}

\author{
DANI C. GUNTHER \\ Washington State University, Pullman, Washington \\ and \\ F. RICHARD FERRARO and TARA KIRCHNER \\ University of North Dakota, Grand Forks, North Dakota
}

\begin{abstract}
The fan effect paradigm was used to investigate the influence of emotional mood state on longterm memory retrieval (Anderson, 1983). Subjects learned target facts embedded in unrelated sentences to a specified criterion and were then given a happy, sad, or neutral mood induction. Mean response times (RTs) and error rates were analyzed in a speeded recognition test in which subjects distinguished between the learned facts and foil facts (foil facts were constructed by recombining the same concepts). A follow-up lexical decision task indicated that mean RT was positively correlated with an increase in the weighted proportion of irrelevant thoughts produced by subjects in an induced sad mood. Results suggest that irrelevant thoughts associated with the sad mood state interfered with more relevant, task-oriented, thoughts and support the notion that sad mood is related to a failure to inhibit irrelevant information.
\end{abstract}

Thoughts vary with a person's emotional mood state, and these thoughts can interfere with other, more relevant, thoughts or behaviors (Ellis \& Ashbrook, 1988; Ellis, Thomas, McFarland, \& Lane, 1985; Ellis, Thomas, \& Rodriguez, 1984; Lubin, 1965; Seibert \& Ellis, 1991a, 1991b). Irrelevant thoughts are attention-diverting and typically affect cognitive performance in a negative fashion (Ellis et al., 1985; Seibert \& Ellis, 1991b). Conversely, relevant thoughts facilitate and focus on successful task performance. Thus, as irrelevant thoughts increase, task performance will decrease (Ellis \& Ashbrook, 1988; Seibert \& Ellis, 1991b).

Working memory theory (Hasher \& Zacks, 1988) contributes to an understanding of how irrelevant thoughts interfere with relevant thoughts. Working memory stores and processes information. During optimal information processing, working memory enables information relevant to comprehension or to successful cognitive performance to co-occur and effectively intermix without interference of irrelevant information (Just \& Carpenter, 1987). Inhibitory attentional mechanisms are intrinsic to working memory, allowing for uninterrupted work to continue. These mechanisms restrict irrelevant thoughts from entering ongoing working memory processing operations. If irrelevant thoughts inadvertently enter processing operations, the inhibitory mechanisms work to suppress the intrusion (Gerard, Zacks, Hasher, \& Radvansky, 1991; Hasher \& Zacks, 1988). However, if the inhibitory mechanisms are weak or inoperative, irrelevant

Correspondence should be addressed to F. R. Ferraro, Department of Psychology, Box 8380, University of North Dakota, Grand Forks. ND 58202-8380 (e-mail: ferraro@badlands.nodak.edu). thoughts may enter the processing operations, the work is interrupted, and performance is impaired. Hence, inhibitory attentional mechanisms are essential for optimal cognitive performance (Hasher \& Zacks, 1988). Thus, if different emotional mood states weaken the inhibitory mechanisms, irrelevant thoughts associated with these mood states would be more likely to interfere with relevant thoughts in working memory.

In the present experiment, we investigated the influence of irrelevant thoughts associated with happy, sad, and neutral mood states on long-term memory retrieval processes. We used Anderson's (1983) fan effect task for the memory manipulation, and we used the lexical decision task (LDT) in a follow-up study. People in experimentally induced sad and happy mood states display longer response times (RTs) on the recognition test than do neutral mood subjects, and we predicted a group $x$ fan interaction for RTs and error rates. Specifically, this interaction should reveal that sad and happy inducedmood subjects should produce longer RTs (and higher error rates) for Fan Level 3-3 sentences than for Fan Level 1-1 sentences. In the follow-up LDT experiment, sad induced-mood subjects would reveal a significant and positive correlation between the weighted proportion of irrelevant thoughts and LDT latencies and error rates.

\section{METHOD}

\section{Subjects}

Sixty-four undergraduates (48 females) participated. All averaged 20 years of age (range $=18-25$ ) and were randomly assigned to one of three conditions: sad, happy, or neutral (control) induced emotional mood state. There were 19 subjects in the sad condition, 23 subjects in the neutral condition, and 22 subjects in the happy condition. The groups were unequal because 10 subjects (there were 74 original sub- 
jects), could not memorize target facts accurately, scored too high on a depression screening instrument, or were over the age of 40 .

\section{Design and Materials}

Psychometric and demographic measures obtained included depression (Ferraro \& Chelminski, 1996), vocabulary (Wechsler, 1981), reading rate (Nelson \& Denny, 1973), age, education, self-rated health, and number(s) and type(s) of medication(s) currently used. These measures took approximately 10-15 minutes to complete.

The memory task required the subjects to learn to a criterion level (correctly answer all the oral recall questions two successive times) a set of target facts embedded in sentences of the form The person [type of professional] performed an activity; for example, The executive cut the apple into six pieces, The teacher found a spot to sunbathe at the beach. Nine characters and nine predicates were used to construct these sentences, and the characters and predicates were combined to form fan levels (Gerard et al., 1991). This activity took varying amounts of time across subjects, although the subjects $(n=5)$ who could not reach criterion in a 75-min time limit were dismissed.

Fan levels are operationally defined as the frequency with which a character or predicate appears on the learning list. The first number of the fan level refers to how many concepts are associated with a character on the learning list; the second, to how many concepts are associated with a predicate on the learning list. The criterion fan levels (character, predicate) are defined as small $(1,1)$, medium $(2,2)$, large $(3,3)$, and filler $(2,3$ and 3,2$)$. There were 3 sentences at the $(1,1)$ level, 3 sentences at the $(2,2)$ level, 3 sentences at the $(2,3)$ level, 3 sentences at the $(3,2)$ level, and 6 sentences at the $(3,3)$ level, for a total of 18 sentences. The list comprised 12 criterion and 6 filler fan level sentences (Gerard et al., 1991).

Seibert and Ellis's (1991a) Self-referencing mood induction procedure was used to induce a temporary mood state for happy, sad, and neutral mood conditions. There are 25 statements presented at 20 -sec intervals in each condition that are emotional for the happy and sad conditions (e.g., The world is full of opportunity and I am taking advantage of it, Ifeel cheated by life) or nonemotional for the neutral condition (e.g., Most oil paintings are done on canvas). The sentences in the happy and sad conditions become progressively more emotionally intense, and the terminology used in these sentences is familiar to most undergraduates. This activity took approximately $8-9 \mathrm{~min}$.

Lubin's Depression Adjective Checklist (DACL; Lubin, 1965) assessed mood induction success. This instrument consists of 21 sadtoned adjectives and 11 happy-toned adjectives (e.g., gloomy or delighted); it is scored by adding the number of sad adjectives checked with the number of happy adjectives not checked. High scores indicate a sadder mood. Although no cut-off scores were employed, we patterned mood induction success after Seibert and Ellis (1991b, pp. 509 510 ). This procedure lasted approximately 1-2 min.

The recognition test was administered on a $386 \mathrm{SX} 25-\mathrm{MHz}$ Gateway 2000 IBM-compatible computer. Micro Experimental Laboratory (MEL) software recorded response times and error rates (Schneider, 1988). The test comprised 18 sentences, nine sentences from the previous learning list and nine false (foil) sentences. Foil sentences were constructed by re-pairing the characters and predicates from the learned sentences (Gerard et al., 1991). This activity took approximately $15-20 \mathrm{~min}$.

\section{Procedure}

The subjects completed a consent form, background questionnaire, and psychometric tests in 15-20 min. Next, a 1-min break occurred, during which the subjects were randomly assigned to the sad, neutral, or happy induced emotional mood states.

The learning phase consisted of a study phase followed by a test phase that was repeated until the subjects learned the target facts on the learning list to the criterion level mentioned earlier. There were $15-\mathrm{sec}$ intervals for each target fact, and the subjects were instructed to memorize the sentence. The sentences were typed on note cards held by the subjects. After the subjects had examined all 18 cards once, they received an oral recall test, which consisted of questioning the subjects about each character and each predicate from the sentences one time (e.g., What did the executive do?, Who sunbathed on the beach?). The questions required the subjects to recall one, two, or three persons or activities, depending on the fan level. A different fan level order and a different recall question order were used randomly for the subjects (Gerard et al., 1991). If subjects answered a question incorrectly, the recall test ended, and the subjects were told the correct answer.

The mood induction phase, following Seibert and Ellis's (1991a) selfreferencing mood induction procedure, consisted of 25 self-referent statements typed on note cards that subjects held in their hands and that were paced at $20-\mathrm{sec}$ intervals. The subjects were instructed that they must experience and feel each statement as it applied to them personally for the mood induction to be successful. They were given written instructions to read before beginning this task. The subjects read each statement once to themselves and once aloud. Those assigned to the happy and sad mood conditions free associated (spoke their feelings), whereas those assigned to the neutral condition continually read the sentences for the remainder of the $25 \mathrm{sec}$. Mood induction success was assessed with Lubin's Depression Adjective Checklist (DACL) Form A, which requires subjects to check off words from a list of 21 negative and 11 positive adjectives that are intended to describe the subjects' feelings at the time.

The speeded recognition phase was computer controlled and included 18 sentences ( 9 previously studied facts, 9 foil facts) that were repeated six times each, resulting in 54 true and 54 false sentences, for a total of 108 randomly distributed sentences (Gerard et al., 1991). The subjects judged as quickly and as accurately as possible whether or not sentences were from the study set. They pressed " 1 " or " 0 " to indicate "yes" or "no," respectively. There was an 18-trial practice block (of which subjects were unaware) before the start of the experimental trials to adapt the subjects to the "yes" and "no" keys. The sentences were displayed one at a time, and they remained in view until the subjects responded. When the subjects made an error, an error message was displayed on the computer monitor. Following the recognition phase, the subjects took Lubin's (1965) DACL Form B (patterned after Form A and scored in the same way) so that we could assess the intensity of the induced emotional mood state. At the end of the experiment, the subjects in the sad and neutral mood state conditions were given 10 happy sentences from Seibert and Ellis's (1991a) self-referencing mood induction procedure to counterbalance any negative feelings still present. The subjects were then debriefed, and any questions that they had were answered.

\section{RESULTS}

\section{Comparability of Groups}

The three groups did not differ on psychometric or demographic variables (all $p \mathrm{~s}>.05$ ), except for reading rate. However, reading rate differences did not influence results. There were no sex differences in any analyses involving demographic variables.

\section{Mood Induction Assessment}

A one-way analysis of variance (ANOVA) revealed that groups differed on Form $\mathrm{A}$ in the predicted direction $\left[F(2,61)=54.99, M S_{\mathrm{e}}=16.98, p<.01\right]$. Mean scores were as follows: $\mathrm{sad}=16.3(S D=6.4)$, neutral $=7.3$ $(S D=3.2)$, happy $=2.9(S D=1.8)$. These are similar to scores obtained by (1) Seibert and Ellis (1991b; sad = 16.8 , neutral $=6.7$, happy $=2.5$ ), and (2) Velten's $(1968)$ mood induction procedure (see Seibert \& Ellis, 1991a). Form B was administered after the recognition test (1520 min later) to ensure that the induced mood states endured testing. Although the happy and sad mood state scores decreased somewhat $(\mathrm{sad}=11.5, S D=6.8$; neutral $=7.6, S D=4.2$; happy $=5.1, S D=3.9$ ), scores still 
differed across the three groups $\left[F(2,61)=8.14, M S_{\mathrm{e}}=\right.$ $25.36, p<.01]$.

\section{Trials to Criterion}

Groups did not differ in learning phase trials to criterion $\left[F(2,61)=1.79, M S_{\mathrm{e}}=5.76, p=.18\right]$. Mean trials to criterion were $4.89(S D=1.76)$ for the sad group, 5.17 $(S D=1.56)$ for the neutral group, and $5.91(S D=2.04)$ for the happy group.

\section{Fan Effects in Recognition}

Subjects whose RTs were 2 SDs above or below their mean, and who scored less than $90 \%$ correct on the recognition test, were excluded. This resulted in less than $2 \%$ of the data being eliminated. Table 1 presents mean RT latency (and percent errors) as a function of group, fan level, and probe type.

Response times. Mean RTs (in milliseconds) were analyzed with a 3 (group: happy, sad, neutral) $\times 3$ (fan: 1-1, 2-2,3-3) $\times 2$ (probe: studied, nonstudied) mixed ANOVA. Group was a between subjects factor; fan and probe were within-subjects factors. There was no main effect of group $\left[F(2,61)=2.28, M S_{\mathrm{e}}=1,001,355.29, p=.11\right]$, but there were main effects of fan $\left[F(2,122)=137.83, M S_{\mathrm{e}}=\right.$ $100,840.44, p<.01]$ and probe $\left[F(2,61)=88.20, M S_{\mathrm{e}}=\right.$ $41,040.16, p<.01]$. Fan and probe interacted $[F(4,122)$ $\left.=18.99, M S_{\mathrm{e}}=62,841.99, p<.01\right]$. The group $\times$ fan, group $\times$ probe, and group $\times$ fan $\times$ probe interactions were not significant $(F \mathrm{~s}<1.39, p \mathrm{~s}>.24)$.

To assess group differences in RT to the studied probe sentences only, a 3 (group) $\times 3$ (fan) mixed ANOVA was performed. There was no main effect of group $[F(2,61)=$ $\left.2.09, M S_{\mathrm{e}}=466,962.85, p=.13\right]$, but there was a main effect of fan $\left[F(4,122)=74.16, M S_{\mathrm{e}}=51,221.08, p<.01\right]$. The group $\times$ fan interaction was marginal $[F(4,122)=$ 2.30, $\left.M S_{\mathrm{e}}=51,221.08, p=.06\right]$.

A third analysis was performed that eliminated the 22 fan level. The 2-2 level was removed because the basic pattern of fan effects on retrieval are that response times should not differ between Fans 1-1 and 2-2, although Fan 1-1 should differ from Fan 3-3 (Anderson, 1983; Gerard et al., 1991). Fan 1-1 and Fan 2-2 performance did not differ $\left[F(1,63)=1.87, M S_{\mathrm{e}}=38,341.50, p=.18\right]$.

Table 1 Mean Reaction Time (in Milliseconds) and Percent Correct as a
Function of Group (Sad, Neutral, Happy), Fan Level (1-1, 2-2, 3-3), and Probe Type (Studied, Unstudied)

\begin{tabular}{|c|c|c|c|c|c|c|}
\hline \multirow[b]{3}{*}{ Fan Level } & \multicolumn{6}{|c|}{ Group } \\
\hline & \multicolumn{2}{|c|}{ Sad } & \multicolumn{2}{|c|}{ Neutral } & \multicolumn{2}{|c|}{ Happy } \\
\hline & $\mathrm{RT}$ & $\%$ & RT & $\%$ & RT & $\%$ \\
\hline \multicolumn{7}{|c|}{ Studied Sentences } \\
\hline $1-1$ & $1,828.6$ & 97.5 & $1,659.6$ & 96.6 & $1,799.1$ & 95.9 \\
\hline $2-2$ & $1,711.5$ & 99.7 & $1,539.3$ & 99.1 & $1,600.3$ & 97.7 \\
\hline $3-3$ & $2,316.4$ & 94.4 & $1,908.0$ & 95.4 & $2,057.5$ & 97.5 \\
\hline \multicolumn{7}{|c|}{ Nonstudied Sentences } \\
\hline $1-1$ & $1,916.6$ & 98.8 & $1,640.0$ & 98.1 & $1,843.4$ & 97.2 \\
\hline $2-2$ & $1,878.0$ & 98.8 & $1,613.1$ & 97.6 & $1,767.1$ & 98.0 \\
\hline $3-3$ & $2,704.9$ & 85.7 & $2,373.2$ & 81.4 & $2,437.5$ & 82.3 \\
\hline
\end{tabular}

Thus, because we are interested in the greatest difference between fan levels, removing Fan 2-2 is acceptable in this situation. A 3 (group) $\times 2$ (fan) ANOVA revealed no main effect of group $\left[F(2,61)=2.13, M S_{\mathrm{e}}=407,586.09\right.$. $p=.1271]$, but there was a main effect of fan $[F(1,61)=$ 70.63, $\left.M S_{\mathrm{e}}=49,473.83, p<.01\right]$. More importantly, group and fan interacted $\left[F(2,61)=3.72, M S_{\mathrm{e}}=49,473.8\right.$, $p<.03]$.

Errors. Percent correct ranged from $81.4 \%$ to $99.7 \%$ correct, and as RT increased, so too did error rate $(r=$ $.83, p<.01)$ indicating no speed-accuracy tradeoff.

\section{Follow-Up Study}

A follow-up study was performed to determine whether the proportion of irrelevant thoughts produced by sad induced-mood subjects was associated with RT increase. No direct evidence of this had been collected in the previous experiment.

Forty-four new subjects ( 36 female) participated. Procedures were identical to those in the previous experiment, except that a sad mood was induced in all subjects. No gender effects existed across psychometric, demographic, or RT performance. Seibert and Ellis (1991b) found that decreases in recall performance were associated with increases in the weighted proportion of irrelevant thoughts produced for sad and happy induced-mood subjects. Performance on the LDT and the fan effect paradigm are similar because RT increases are thought to reflect increases in the time taken to retrieve information (i.e., Is this letter string a word? Is this fact related to the target?). Increases in the information retrieval process would also produce increases in error rate.

Subjects had a mean DACL (Form A) score of 14.5 ( $S D=4.9$ ), which is similar to the sad induced-mood subjects' DACL scores reported in the previous experiment $(M=16.3, t=1.22, p>.05)$ and to the findings of Seibert and Ellis (1991, $M=16.8)$. Next, subjects participated in a $10-$ min LDT ( 120 words and 120 pseudowords taken from Kellas, Ferraro, \& Simpson, 1988). Using MEL software, stimuli were displayed for $1,500 \mathrm{msec}$ or until subjects responded. If the letter string was a word (or pseudoword), the subjects pressed the " 1 " (or " 0 ") key with the left- (or right-) hand index finger. No speedaccuracy tradeoff existed, and mean LDT RTs increased as error rates increased $[r(42)=.91, p<.01]$.

Next, the subjects performed the delayed thought listing task (Seibert \& Ellis, 1991b) immediately after the completion of the LDT. The subjects were given a sheet of paper with 10 spaces on it and were instructed to write down all the thoughts that came to mind during the LDT. If the subject completed all 10 spaces before $2.5 \mathrm{~min}$ had expired, they listed further thoughts on the paper. When the time had expired, the subjects rated each listed thought in three ways. First, they rated their thoughts as either hindering (task irrelevant or nonfacilitative) or helping (task relevant or facilitative) their LDT performance. Second, they estimated the frequency of the thought's occurrence, using a not very frequent ( $1 \mathrm{pt}$.), frequent ( 2 pts.), or very frequent ( 3 pts.) scale. Third, 
they rated the intensity of each thought produced, indicating whether the thought had a weak (1 pt.), somewhat weak ( 2 pts.), somewhat strong ( 3 pts.), or strong ( 4 pts.) effect on their successful LDT performance.

We weighted the subjects' irrelevant thoughts to account for judged intensity and judged frequency (Seibert \& Ellis, 1991). Each thought's frequency rating (1-3) was arbitrarily multiplied by its intensity rating (1-4), resulting in a weighted score for each thought produced (range $=1-12$ ). Weighted scores for each subject were then summed in accordance with whether they were judged relevant (helpful) or irrelevant (hindered), and the sum of the weighted irrelevant thoughts was divided by the total of all of weighted scores (relevant + irrelevant). Consider Subject 1 as an example. She listed 12 thoughts, 9 of which hindered LDT performance. Her rated frequency $X$ intensity sum for the 9 hindered (irrelevant) thoughts totaled 80 , while her rated frequency $\times$ intensity sum for nonhindered (relevant) thoughts totaled 24 . Her weighted proportion of irrelevant thoughts was equal to $.77[.77=$ $(80) /(80+24)]$. Across subjects, the mean weighted proportion of irrelevant thoughts was $.82(S D=.21$, range $=$ $.06-1.00$ ), which is comparable to data reported by Seibert and Ellis (1991). To determine whether the weighted proportion of irrelevant thoughts was associated with increases in LDT RT, a correlation coefficient was calculated by using each subjects' mean proportion of weighted irrelevant thoughts and their mean overall LDT RT (collapsed across words and pseudowords). This resulted in $r(42)=.67, p<.01$, supporting the conclusion that longer RTs result from irrelevant thoughts associated with the sad mood. LDT error rate and proportion of weighted irrelevant thoughts produced were also associated $[r(42)$ $=.57, p<.01]$.

\section{DISCUSSION}

The goal of the present experiments was to investigate the influence of irrelevant thoughts associated with happy and sad emotional mood states on retrieval of relevant information in long-term memory.

Results confirmed the hypothesis that the sad induced-mood group would show slower RTs than would the neutral mood group on the speeded recognition test. This pattern of results corroborates Ellis et al. (1985), who showed evidence of sad detrimental mood effects on retrieval processes in long-term memory. An important similarity between the Ellis et al. (1985) study and the present study can be seen in the type of information subjects retrieved from memory. In both experiments, subjects learned target information embedded in unrelated sentences and then had to either recognize or recall the target facts during a subsequent test - a relatively demanding task for encoding and retrieval processes. Thus, the notion that the type of material learned in cognition-emotion investigations can influence the outcome of the studies is supported (Ellis \& Ashbrook, 1988).

The results of the sad mood state effects on memory can be explained by the working memory theory of inhibitory attentional mechanism. Irrelevant thoughts associated with emotional mood states can disrupt cognitive task performance and divert attention (Seibert $\&$ Ellis, 1991a). In this light, irrelevant thoughts associated with a sad mood state can be seen as interfering with thoughts relevant to the task at hand by weakening or impairing inhibitory attentional mechanism in working memory (Hasher \& Zacks, 1988). The present results lend support to this notion by showing that subjects in the sad induced mood state showed greater interference effects of irrelevant thoughts on relevant information in working memory than did subjects in the neutral mood state on the recognition test.

In summary, we have provided evidence from two separate experiments, which, when taken together, links the increase in the production of irrelevant thoughts to the slowing of RTs of subjects in whom a sad mood has been induced (Seibert \& Ellis, 1991a, 1991b). These results confirm that basic cognitive performance is adversely affected (and increases in RTs, error rates, and the proportion of irrelevant thoughts are produced) during a sad emotional mood state.

\section{REFERENCES}

ANDERSON, J. R. (1983). The architecture of cognition. Cambridge, MA: Harvard University Press.

Ellis, H. C., \& AshBrooK, P. W. (1988). Resource allocation model of the effects of depressed mood states on memory. In K. Fiedler \& J. Forgas (Eds.), Affect, cognition, and social behavior (pp. 25-43). Toronto: Hogrefe.

Ellis, H. C., Thomas, R. L., McFarland, A. D., \& Lane, J. W. (1985). Emotional mood states and retrieval in episodic memory. Journal of Experimental Psychology, 11, 363-370.

Ellis, H. C., Thomas, R. L., \& Rodriguez, I. A. (1984). Emotional mood states and memory: Elaborative encoding, semantic processing, and cognitive effort. Journal of Experimental Psychology: Learning, Memory, \& Cognition, 10, 470-482.

Ferraro, F. R., \& Chelminski, I. (1996). Preliminary normative data on the Geriatric Depression Scale-Short Form (GDS-SF) in a young adult sample. Journal of Clinical Psychology, 52, 443-447.

Gerard, L., Zacks, R. T., Hasher, L., \& Radvansky, G. A. (1991). Age deficits in retrieval: The fan effect. Journals of Gerontology, 46, P131-P136.

HASHER, L., \& ZACKS, R. T. (1988). Working memory, comprehension, and aging: A review and a new view. In G. H. Bower (Ed.), The psychology of learning and motivation (Vol. 22, pp. 193-225). New York: Academic Press.

JUST, M. A., \& CARPENTER, P. A. (1987). The psychology of reading and language comprehension. Boston: Allyn \& Bacon.

Kellas, G., Ferraro, F. R., \& Simpson, G. B. (1988). Lexical ambiguity and the timecourse of resource allocation in word recognition. Journal of Experimental Psychology: Human Perception \& Performance, 14, 601-609.

LUBIN, B. (1965). Adjective checklists for measurement of depression. Archives of General Psychiatry, 12, 57-62.

Nelson, M. J., \& Denny, E. C. (1973). The Nelson-Denny Reading Test-Form D. Boston, MA: Houghton Mifflin Co.

SCHNEIDER, W. (1988). MEL Experimental Laboratory: An integrated system for IBM PC compatibles. Behavior Research Methods. Instruments, \& Computers, 20, 206-217.

SEIBERT, P. S., \& Ellis, H. C. (1991a). A convenient self-referencing mood induction procedure. Bulletin of the Psychonomic Society, 29, $121-124$.

SEIBERT, P. S., \& ELLis, H. C. (1991b). Irrelevant thoughts, emotional mood states, and cognitive task performance. Memory \& Cognition, $19,507-513$

VELTEN, E. (1968). A laboratory test for induction of mood states. Behavior Research \& Therapy, 6, 473-482.

WeCHSLER, D. J. (1981). Manual: Wechsler Adult Intelligence Scale: Vocabulary Test (WAIS-R). New York: Psychological Corp.

(Manuscript received July 13, 1995; revision accepted for publication June 12, 1996.) 